\title{
Provision of free nicotine replacement therapy to parental smokers in the pediatric emergency setting
}

\author{
E. Melinda Mahabee-Gittens ${ }^{1}$, Ashley L. Merianos², Meredith E. Tabangin ${ }^{3}$, Lara Stone', Judith S. Gordon ${ }^{4}$, Jane C. Khoury ${ }^{3}$
}

\begin{abstract}
INTRODUCtION Although the administration of free Nicotine Replacement Therapy (NRT) is effective in helping smokers quit, the feasibility, acceptability and safety of this practice have not been examined in the emergency setting of the pediatric emergency department (PED) or urgent care (UC). We examined the characteristics of parental smokers who were interested and eligible for free NRT during their child's emergency visit and the uptake, usage, and associated side effects of NRT use.

METHODS We analyzed data from 377 parental smokers who were randomized to receive cessation counseling and free NRT as part of an emergency visit-based randomized controlled trial. Parents interested in NRT were screened for medical contraindications; eligible parents were given a 6-week supply of NRT patches or lozenges during their child's emergency visit and offered another supply 6 weeks later. We conducted Wilcoxon rank-sum tests and chi-squared tests to address our main study objective.

RESULTS The majority of parents were female (87.5\%), non-Hispanic Black (52.5\%), and mean (SD) age was 33.1 (8.2) years. A total of $252(66.8 \%)$ parents were interested in receiving NRT. Compared to uninterested parents, interested parents were more likely to: be older [33.6 (8.2) vs 31.9 (8.2), years]; be non-Hispanic Black (54.0\% vs 49.6\%); have older children [5.5 (5.0) vs 4.2 (4.6)]; have a higher readiness to quit [7.0 (2.4) vs $5.2(2.6)$ ]; and have a child being evaluated in UC compared to the PED (72.4\% vs $56.5 \%$ ). A total of $53(21 \%)$ interested parents had $>1$ NRT contraindications. At 6 weeks, 94 (79.0\%) parents reported some $\geq$ NRT usage and $50(53.2 \%)$ requested an additional 6-week supply. There were no serious adverse events and 5 (5.3\%) reported minor side effects.

CONCLUSIONS Parental smokers in the emergency setting are interested in receiving free NRT, the majority use it, and use is not associated with adverse side effects. The emergency visit may be an optimal time to offer NRT to parental smokers.
\end{abstract}

\begin{abstract}
AFFILIATION
1 Division of Emergency Medicine, Cincinnati Children's Hospital Medical Center, University of Cincinnati College of Medicine,

Cincinnati, United States

2 School of Human Services,

University of Cincinnati, Cincinnati, United States

3 Division of Biostatistics and Epidemiology, Cincinnati Children's Hospital Medical Center, University of Cincinnati College of Medicine, Cincinnati, United States

4 College of Nursing, University of Arizona, Tucson, United States
\end{abstract}

\section{CORRESPONDENCE TO}

E. Melinda Mahabee-Gittens. Division of Emergency Medicine, Cincinnati Children's Hospital Medical Center, 3333 Burnet Avenue, MLC 2008, Cincinnati, $\mathrm{OH}, 45229$, United States. E-mail: Melinda.Mahabee-Gittens@cchmc.org

\section{KEYWORDS}

tobacco, nicotine dependence, nicotine replacement therapy, tobacco-use cessation

Received: 3 February 2020

Revised: 6 March 2020

Accepted: 17 March 2020

\section{INTRODUCTION}

Pediatric practitioners are encouraged to use every healthcare visit as an opportunity to screen parents about tobacco use and counsel parental smokers to quit smoking to improve their child's health ${ }^{1-4}$. Although rates of smoking cessation counseling in pediatric primary care settings are increasing, rates of recommending or prescribing pharmacological cessation therapy remain low at $15 \%$ and $3 \%$, respectively ${ }^{5}$. Reasons for low prescription rates by pediatric providers include concerns about lack of knowledge and scope of practice, due to feeling uncomfortable in prescribing medications for parents who are not their patients ${ }^{5-7}$. Nevertheless, the American Academy of Pediatrics strongly encourages pediatric practitioners to prescribe pharmacological 
cessation medications in conjunction with counseling during children's healthcare visits to help parents quit smoking ${ }^{2,3}$.

The provision or prescription and use of Nicotine Replacement Therapy (NRT) is effective in helping adult smokers quit smoking ${ }^{8}$. NRT helps to increase abstinence by temporarily replacing much of the nicotine obtained from cigarettes ${ }^{1,8-11}$. NRT has an excellent safety profile with rare cases of associated serious adverse events ${ }^{8}$. NRT is available overthe-counter and pharmacists are able to prescribe NRT in some states ${ }^{12}$. NRT is also covered by most insurance plans, including Medicaid. Thus, the use of NRT is strongly encouraged in cessation attempts. NRT has been administered to lowincome smokers who present to the adult emergency department (ED) as part of cessation interventions with high rates of acceptance and some success in helping smokers quit ${ }^{9,13-16}$. To our knowledge, no research studies based on pediatric ED (PED) or pediatric urgent care (UC) settings have examined the direct administration of free NRT to parents as part of a cessation intervention during their child's emergency visit. To fill this gap, we recently conducted a large randomized controlled trial (RCT) called Healthy Families (NCT02531594), which included cessation counseling and the provision of free NRT to parental smokers during their child's emergency visit. In this study, our objective was to present the results on the number and characteristics of parents who were interested in free NRT compared to those who were not. For parents interested in NRT, we assessed their eligibility to receive a 6 -week supply of free NRT. After 6 weeks, we followed up with parents who received free NRT to assess their uptake, usage patterns, any associated side effects, and interest in receiving another 6-week NRT supply. The results of this study will inform future researchers and practitioners who are planning to administer free NRT to adult smokers in the pediatric emergency setting.

\section{METHODS}

\section{Design, participants and IRB approval}

We analyzed data from the Healthy Families RCT, a tobacco cessation intervention for parental smokers who presented to the PED or UC of a Midwestern Children's Hospital from April 2016 through May
2019. Participants in Healthy Families were parents/ legal guardians who were current smokers and seeking pediatric care for their child 0-17 years of age. A total of 750 parents were recruited and randomly assigned to either the Screening, Intervention, Referral to Treatment (SBIRT) condition $(n=377)$ or to an attention control condition $(n=373)$. Only data from the 377 parental smokers in the SBIRT condition were analyzed in order to address the study objectives. Healthy Families study details are described elsewhere ${ }^{17}$, but briefly, parents randomized into the SBIRT condition received face-to-face, tailored smoking cessation counseling that focused on the child's illness and the option to receive 6 weeks of free NRT (patch or lozenge) if they agreed to set a quit date within 14 days of their child's emergency visit. Parents were followed up in their homes 6 weeks after the emergency visit and offered an additional 6-week supply of NRT if they reported using at least $80 \%$ of the initial 6 -week NRT supply and remained interested and eligible to use NRT. The SBIRT interventionists were either clinical research coordinators or social workers who had, at minimum, a college degree and were trained as Tobacco Treatment Specialists at an accredited Tobacco Treatment Specialist Training program (e.g. The ACT Center for Tobacco Treatment, Education and Research).

Results of the cessation outcomes of parents who used NRT in combination with the rest of the SBIRT will be presented elsewhere. Healthy Families was approved by our hospital's IRB and participant consent was obtained. Since the adult participants were not patients at our hospital, there were concerns regarding dispensing of NRT in this setting. Therefore, we had to seek approval from leadership in clinical research and from the Pharmacy Department to administer free NRT and approval on the criteria used to determine which participants were eligible for NRT. As part of our IRB protocol, we were required to have the SBIRT interventionist call the principal investigator (first author) to review a checklist of contraindications prior to administering NRT. The contraindications were more stringent than recommended $^{18,19}$ due to initial concerns about NRT administration in our setting. After approximately one year of this practice, our IRB allowed us to amend our protocol and the interventionist only 
had to call if there were any concerns or questions about responses on the checklist. We were cognizant that combination NRT (e.g. nicotine patches plus lozenges) would likely result in higher long-term quit rates than single NRT for some participants ${ }^{10}$, but due to the novelty of NRT distribution in our setting for this study, we were allowed to provide only single modality NRT to participants.

\section{Assessments}

During the child's emergency visit, parents completed assessments that included: sociodemographic factors, financial strain (three items, each 1-5 range; items were added and means reported $)^{20}$, smoking behavior including nicotine dependence and prior quit attempts, readiness to quit smoking as measured by the Contemplation ladder (range: $0-10)^{21}$, heavy smoking index (HSI) to assess level of nicotine dependence ${ }^{22}$, and whether the child was being evaluated in the PED or UC.

After completing the baseline assessments, all parents received smoking cessation counseling, which included information on NRT use ${ }^{17}$. They were asked if they were interested in NRT, and if so, if they were willing to set a quit date within the next 14 days. All interested parents willing to set a quit date were subsequently asked if they preferred NRT patches or lozenges. They were then screened for eligibility for their first choice of NRT by asking about any potential medical contraindications. If parents were ineligible for their first choice, they were screened for their second choice. Eligible parents with no contraindications who received NRT were told to report any potential NRT-related side effects of concern at any time to study staff. We grouped parents based on their interest in receiving NRT (interested, not interested).

\section{Statistical analysis}

Data were collected within a REDCap ${ }^{\circledR}$ database, where data entry branching and limited checking were in place to aid with accuracy and completeness of the information. The majority of the data were electronically entered by the enrolled parent. The data used were exported to SAS ${ }^{\circledR}$ version 9.4 (SAS Institute, Cary, NC) for analysis. Prior to analysis, all data were checked for outliers and consistency. We calculated means and associated standard deviations
(SDs), medians with 25 th and 75 th percentiles to show the interquartile range (IQR), and minimum and maximum values to depict the range. Continuous variables were compared between the 'interested' in NRT and 'not interested' in NRT groups using Wilcoxon rank-sum test, due to the general skewed nature of the data. Categorical variables were compared between the two NRT interest groups using chi-squared tests. A p-value $<0.05$ was considered statistically significant.

\section{RESULTS}

The majority of parents were female $(87.5 \%)$, nonHispanic Black (52.5\%), with a mean age (SD) of 33.1 (8.2) years, low income with $61.3 \%$ reporting an annual income of $<\$ 15000$. Parents had a low level of nicotine dependence on average $(\mathrm{HSI}<4)^{22}$ with a mean HSI (SD) of 2.17 (1.38).

A total of $252(66.8 \%)$ parents were willing to set a quit date and interested in receiving NRT. Compared to parents who were not interested in NRT $(n=125$; $33.2 \%)$, interested parents were more likely to: be older with mean (SD) parent age 33.6 (8.2) vs 31.9 (8.2) years; have older mean (SD) child age 5.5 (5.0) vs 4.2 (4.6) years; be non-Hispanic Black ( $54 \%$ vs $49.6 \%$ ), and have higher motivation to quit [mean (SD) $7.0(2.4)$ vs. $5.2(2.6)]$. Of the parents with a child being evaluated in UC (65.2\%), the majority were interested in NRT (70.6\% vs $54.5 \%$ ) (Table 1). Additionally, more parents with a child being evaluated in UG were interested in NRT compared to parents with a child being evaluated in the PED ( $72.4 \%$ compared to $56.5 \%$; $\mathrm{p}=0.002$ ).

\section{Screening for NRT eligibility}

After assessing willingness to set a quit date in 14 days and interest in receiving free NRT, of the 252 parents that were asked, 155 preferred NRT patches and 97 preferred lozenges. Parents were then screened with a modified version of the U.S. Food and Drug Administration (FDA)-approved contraindications for $\mathrm{NRT}^{18,19}$ created for this study. We used the strictest criteria for NRT approval given the literature that reports that pediatricians are uncomfortable prescribing cessation medications to adults who are not their direct patients ${ }^{5}$. Table 2 gives the checklist and frequencies of contraindications for NRT. A total of $53(21.0 \%)$ interested parents were excluded 
Table 1. Parent characteristics overall and by interest in setting a quit date and receiving free NRT

\begin{tabular}{|c|c|c|c|c|}
\hline Characteristics & $\begin{array}{l}\text { Overall } \\
(\mathrm{N}-377)\end{array}$ & $\begin{array}{l}\text { Interested in NRT } \\
\qquad(\mathrm{N}-252)\end{array}$ & $\begin{array}{l}\text { Not interested } \\
\text { in NRT } \\
(\mathrm{N}-125)\end{array}$ & $p^{*}$ \\
\hline Parent age (years) & & & & 0.02 \\
\hline Mean (SD) & $33.1(8.2)$ & $33.6(8.2)$ & $31.9(8.2)$ & \\
\hline Median (IQR) & $31.0(27.0-37.0)$ & $32.0(28.0-38.0)$ & $30.0(26.0-35.5)$ & \\
\hline Range & $20.0-64.0$ & $20.0-64.0$ & $20.0-64.0$ & \\
\hline Child age (years) & & & & 0.008 \\
\hline Mean (SD) & $5.1(4.9)$ & $5.5(5.0)$ & $4.2(4.6)$ & \\
\hline Median (IOR) & $3.3(1.1-8.1)$ & $3.9(1.3-9.0)$ & $2.2(0.7-6.6)$ & \\
\hline Range & $0-17.8$ & $0-17.8$ & $0-17.3$ & \\
\hline \multicolumn{5}{|l|}{ Parent sex, n (\%) } \\
\hline Female & $330(87.5)$ & $215(85.3)$ & $115(92.0)$ & 0.06 \\
\hline Parent race, n (\%) & & & & 0.01 \\
\hline Black, non-Hispanic & $198(52.5)$ & $136(54.0)$ & $62(49.6)$ & \\
\hline White, non-Hispanic & 149 (39.5) & 93 (36.9) & $56(44.8)$ & \\
\hline Hispanic & $8(2.1)$ & $3(1.2)$ & $5(4.0)$ & \\
\hline Other & $22(5.8)$ & $20(7.9)$ & $2(1.6)$ & \\
\hline Insurance type, n (\%) & & & & 0.65 \\
\hline Public/self-pay & $324(85.9)$ & $218(88.5)$ & $106(84.8)$ & \\
\hline \multicolumn{5}{|l|}{ Commercial } \\
\hline Income, n (\%) & & & & 0.74 \\
\hline$\leq 5000$ & $144(38.2)$ & $98(38.9)$ & $46(36.8)$ & \\
\hline $5001-15000$ & $87(23.1)$ & $53(21.0)$ & $34(27.2)$ & \\
\hline $15001-30000$ & $88(23.3)$ & $62(24.6)$ & $26(20.8)$ & \\
\hline $30001-50000$ & $40(10.6)$ & $28(11.1)$ & $12(9.6)$ & \\
\hline$>50001$ US\$ & $18(4.8)$ & $11(4.4)$ & $7(5.6)$ & \\
\hline Financial strain & & & & 0.07 \\
\hline Mean (SD) & $2.46(1.10)$ & $2.53(1.10)$ & $2.33(1.10)$ & \\
\hline Median (IOR) & $2.33(1.67-3.33)$ & $2.33(1.67-3.33)$ & $2.00(1.33-3.00)$ & \\
\hline Range & $1.00-5.00$ & $1.00-5.00$ & $1.00-5.00$ & \\
\hline Unemployed, n/N (\%) & $162 / 375(43.2)$ & $103 / 250(41.2)$ & $59 / 125(47.2)$ & 0.27 \\
\hline Education, n (\%) & & & & 0.23 \\
\hline Less than HS & 67 (17.8) & $39(15.5)$ & $28(22.4)$ & \\
\hline Completed HS & $153(40.6)$ & $105(41.7)$ & $48(38.4)$ & \\
\hline Vocational school & $157(41.6)$ & $108(42.9)$ & 49 (39.2) & \\
\hline \multicolumn{5}{|l|}{ Some College and beyond } \\
\hline Cigarettes/day & & & & 0.89 \\
\hline Mean (SD) & $11.5(7.1)$ & $11.3(6.6)$ & $11.8(7.9)$ & \\
\hline Median (IOR) & $10.0(6.0-15.0)$ & $10.0(6.0-15.0)$ & $10.0(6.0-15.0)$ & \\
\hline Range & $1.0-40.0$ & $1.0-40.0$ & $2.0-40.0$ & \\
\hline Prior quit attempts, n (\%) & & & & 0.20 \\
\hline 0 & $116(30.8)$ & $71(28.2)$ & $45(36.0)$ & \\
\hline 1 & $78(20.7)$ & $51(20.2)$ & $27(21.6)$ & \\
\hline$\geq 2$ & $183(48.5)$ & $130(51.6)$ & $53(42.4)$ & \\
\hline
\end{tabular}


Table 1. Continued

\begin{tabular}{|c|c|c|c|c|}
\hline Characteristics & $\begin{array}{l}\text { Overall } \\
(\mathrm{N} 377)\end{array}$ & $\begin{array}{c}\text { Interested in NRT } \\
\text { (N 252) }\end{array}$ & $\begin{array}{l}\text { Not interested } \\
\text { in NRT } \\
(\mathrm{N}=125)\end{array}$ & $p^{*}$ \\
\hline HSI & & & & 0.58 \\
\hline Mean (SD) & $2.17(1.38)$ & $2.19(1.35)$ & $2.11(1.44)$ & \\
\hline Median (IQR) & $2.0(1.0-3.0)$ & $2.0(1.0-3.0)$ & $2.0(1.0-3.0)$ & \\
\hline Range & $0.0-6.0$ & $0.0-6.0$ & $0.0-6.0$ & \\
\hline Readiness to quit & & & & $<0.001$ \\
\hline Mean (SD) & $6.40(2.59)$ & $6.98(2.38)$ & $5.23(2.61)$ & \\
\hline Median (IQR) & $6.0(5.0-8.0)$ & $7.0(6.0-9.0)$ & $5.0(4.0-7.0)$ & \\
\hline Range & $0.0-10.0$ & $0.0-10.0$ & $0.0-10.0$ & \\
\hline Patient in UC, $\mathrm{n}(\%)$ & $246(65.3)$ & $178(70.6)$ & $68(54.4)$ & 0.002 \\
\hline
\end{tabular}

NRT: nicotine replacement therapy. SD: standard deviation. IQR: interquartile range. HS: high school. HIS: heavy smoking index. UC: urgent care. *Wilcoxon rank-sum or chisquared test, as appropriate.

Table 2. Contraindication checklist and frequency of NRT ineligibility for first product choice by interested parents $(\mathrm{N}=252)$

\begin{tabular}{|c|c|c|}
\hline Potential contraindications & $\begin{array}{l}\text { NRT patches } \\
\begin{array}{c}(\mathbf{N}=155) \\
\text { n }(\%)\end{array}\end{array}$ & $\begin{array}{c}\text { NRT } \\
\text { loyenges } \\
(\mathrm{N}=97) \\
\text { n (\%) }\end{array}$ \\
\hline Pregnancy & $3(1.9)$ & 0 \\
\hline Adhesion allergy ${ }^{a}$ & $3(1.9)$ & NA \\
\hline Jaw problems or TMJ ${ }^{b}$ & NA & $3(3.1)$ \\
\hline Mouth or tongue problems ${ }^{b}$ & NA & 0 \\
\hline \multicolumn{3}{|l|}{ Cardiac-related issues } \\
\hline Heart disease & $3(1.9)$ & $1(1)$ \\
\hline Arrhythmias & $1(0.6)$ & 0 \\
\hline Chest pain/angina & $5(3.2)$ & 0 \\
\hline History of heart attack & $4(2.6)$ & 0 \\
\hline Psoriasis or dermatitis ${ }^{a}$ & $1(0.6)$ & 0 \\
\hline Active peptic ulcers or esophagitis & 0 & 0 \\
\hline Severe kidney problems & $1(0.6)$ & 0 \\
\hline Hypertension $^{c}$ & $16(10.3)$ & $4(4.1)$ \\
\hline Hyperthyroidism & 0 & $1(1)$ \\
\hline Pheochromocytoma & 0 & 0 \\
\hline Diabetes mellitus & $1(0.6)$ & $6(6.2)$ \\
\hline
\end{tabular}

from receiving NRT because they had at least one contraindication with the top two being hypertension $(\mathrm{n}=20 ; 7.9 \%)$ and cardiac-related issues $(\mathrm{n}=14 ; 5.6 \%)$.

\section{Dosing for NRT}

Parents who were screened as eligible for NRT and who preferred NRT patches received a 6-week supply of the $21 \mathrm{mg}$ patch if they smoked $>20$ cigarettes per day; otherwise they were given the $14 \mathrm{mg}$ patch. Eligible parents who preferred NRT lozenges received a 6-week supply of the $4 \mathrm{mg}$ lozenge if they smoked their first cigarette within 30 minutes of waking up; otherwise they were given the $2 \mathrm{mg}$ lozenge.

\section{Receipt and subsequent use of NRT}

Of the 199 NRT eligible parents, $100 \%$ received NRT during their child's emergency visit. A total of $129(65 \%)$ received patches: 68 (53\%) received the $21 \mathrm{mg}$ patch and the remainder received the $14 \mathrm{mg}$ patch. Seventy (35\%) received lozenges: 48 (69\%) received the $4 \mathrm{mg}$ lozenge and the remainder received the $2 \mathrm{mg}$ lozenge.

Home visits were successfully conducted on 119 (59.8\%) parents who received NRT during their child's emergency visit. Of those, 94 (79.0\%) reported they used at least some of the NRT and 65 $(54.6 \%)$ used at least $80 \%$ of their original supply, and $50(53.2 \%)$ requested an additional 6 -week supply. A total of 22 (44\%) participants requested to have their NRT modality changed at the 6 -week time point: 13 changed from the NRT patch to lozenge, and 9 changed from the NRT lozenge to patch. No serious adverse events were reported among parents. Only 5/94 (5.3\%) participants reported NRT patchrelated minor side effects: rash $(n=3)$, pruritis $(n=1)$, and nausea $(n=1)$. 


\section{DISCUSSION}

Despite the knowledge that NRT is effective in increasing abstinence in smokers, few pediatric healthcare providers prescribe NRT to parents ${ }^{5-7}$. This study is the first to provide free NRT to parental smokers during their child's (and not the parents') emergency visit. In a previous PED-based project, we conducted a similar study in which parents were given a voucher for a free 2 -week NRT supply that they had to redeem at the hospital pharmacy ${ }^{23}$. Although $92 \%$ of eligible parents expressed interest in receiving the voucher, only $47 \%$ of eligible parents redeemed the voucher and usage rate was not obtained. In contrast, only $67 \%$ of parents in the present study were interested but $100 \%$ of these parents who had no contraindications received NRT during their child's emergency and $79 \%$ reported some use. Since NRT is the preferred choice of cessation medication of many smokers ${ }^{24,25}$, our findings suggest that the provision of free NRT is acceptable to adult smokers in the emergency setting. Research in the adult in-patient setting has found that the provision of free NRT results in increased rates of subsequent use during hospitalization and after discharge ${ }^{26,27}$. The high rate of NRT usage after the emergency visit reported in our study parallels these findings and is encouraging.

Previous studies have provided free NRT to smokers in the adult ED. Overall, these studies have demonstrated increased periods of abstinence and increased quit attempts in those who were given $\mathrm{NRT}^{9,13-16}$. Before evaluating the success of NRT on abstinence among parental smokers in the emergency setting, pediatric healthcare providers and researchers need guidance on which parents may be more receptive to receiving NRT and the potential associated side effects of NRT. This knowledge may potentially increase providers' confidence and comfort in providing NRT to adults who are not their patients. Based on our results, parents in the UC setting and parents who are older, Black, and more motivated to quit, may be more receptive to receiving free NRT. While the differences in parent and child age were statistically significant, the differences may not be clinically meaningful as the relatively small actual difference in age may not help to guide decisions on which parents are likely to be interested in NRT. However, the finding that parents who had a child in the UC were more interested in NRT, compared to parents who had a child in the PED, was more robust and may help to guide the provision of NRT in a clinical setting. A possible explanation for this is that when a child is in the PED, parents may be more concerned about their child's illness than they are about quitting smoking compared to when a child is in the UC and not as sick. The PED setting may also be larger, busier and more overwhelming to parents whereas the UC environment may be perceived as more similar to an outpatient clinic. This finding suggests that the administration of free NRT may be received better in the UC setting, but further research to explore the reasons for interest in NRT is warranted. Similar to other research ${ }^{24,28,29}$, we also found that parents who were Black or more motivated to quit were more receptive to cessation efforts. These findings are clinically relevant to decision-making about NRT. Clinicians and researchers may want to focus their efforts on interventions for Black smokers and/or develop methods for assessing motivation to quit that fit into the PED and UC work flows. Although other research has shown that lower income groups were not receptive to cessation interventions ${ }^{24,30-32}$, we did not observe any differences in interest in NRT with respect to either income or education. Moreover, we did not observe higher rates of interest in parents with higher nicotine dependence as seen in other studies $^{24,33}$, but this is most likely due to the overall lower levels of nicotine dependence observed in our sample.

Using strict criteria to identify parents with potential contraindications to NRT, we found that $21 \%$ were ineligible. However, certain criteria such as hypertension are usually not considered contraindications in real world adult settings ${ }^{18,19,34}$. Thus, more parents would likely be eligible to receive NRT in the emergency setting. For example, if we eliminated hypertension as a contraindication, then only $13 \%$ of parents would have been considered ineligible for NRT. Additionally, similar to work that reports low rates of side effects of $\mathrm{NRT}^{8,35}$, parents in this study reported only minor expected side effects. The lack of serious side effects in screened participants underscores the safety of providing NRT (which is available over-the-counter) in the emergency setting. 


\section{Limitations}

There are limitations that should be considered when interpreting our results. The study sample was drawn from a population of smokers who presented with their children to a tertiary care PED or UC at a single Midwestern children's hospital, which limits generalizability. This population was predominantly from households with low socioeconomic status, which also limits generalizability. We were unable to follow up with all parents who received NRT at 6 weeks so rates of subsequent usage may have been lower or higher than reported.

\section{CONCLUSIONS}

This study provides important information on the interest of receiving free NRT, the feasibility of providing NRT, and the safety of using NRT among parental smokers in the emergency setting. We found that parental smokers who were older, had older children, were non-Hispanic Black, had a higher readiness to quit, and who had a child being evaluated in the UC, as opposed to the PED, were more interested in receiving NRT. Since all parental smokers need to be assisted in quitting smoking, finding ways to engage all smokers in quit attempts at every pediatric healthcare visit should be further examined and prioritized in future studies.

\section{REFERENCES}

1. Jenssen BP, Wilson KM. Tobacco Control and Treatment for the Pediatric Clinician: Practice, Policy, and Research Updates. Acad Pediatr. 2017;17(3):233-242. doi:10.1016/j.acap.2016.12.010

2. Farber HJ, Walley SC, Groner JA, Nelson KE, Section on Tobacco C. Clinical Practice Policy to Protect Children From Tobacco, Nicotine, and Tobacco Smoke. Pediatrics. 2015;136(5):1008-1017. doi:10.1542/peds.2015-3108

3. Farber HJ, Groner J, Walley S, Nelson K, Section On Tobacco C. Protecting Children From Tobacco, Nicotine, and Tobacco Smoke. Pediatrics. 2015;136(5):e14391467. doi:10.1542/peds.2015-3110

4. Marbin JN, Gribben V. Tobacco Use as a Health Disparity: What Can Pediatric Clinicians Do? Children. 2019;6(2):31. doi:10.3390/children6020031

5. McMillen R, O'Connor KG, Groner J, Tanski S, Park ER, Klein JD. Changes and Factors Associated With Tobacco Counseling: Results From the AAP Periodic Survey. Acad Pediatr. 2017;17(5):504-514. doi:10.1016/j.acap.2017.01.002

6. Committee on Environmental Health, Committee on Substance Abuse, Committee on Adolescence, Committee on Native American Child. Tobacco use: a pediatric disease. Pediatrics. 2009;124(5):1474-1487. doi:10.1542/peds.2009-2114

7. Torok MR, Lowary M, Ziniel SI, et al. Perceptions of Parental Tobacco Dependence Treatment Among a Children's Hospital Staff. Hosp Pediatr. 2018;8(11):724728. doi:10.1542/hpeds.2018-0009

8. Hartmann-Boyce J, Chepkin SC, Ye W, Bullen C, Lancaster T. Nicotine replacement therapy versus control for smoking cessation. Cochrane Database Syst Rev. 2018;5:CD000146. doi:10.1002/14651858.cd000146.pub5

9. Pelletier JH, Strout TD, Baumann MR. A systematic review of smoking cessation interventions in the emergency setting. Am J Emerg Med. 2014;32(7):713724. doi:10.1016/j.ajem.2014.03.042

10. Lindson N, Chepkin SC, Ye W, Fanshawe TR, Bullen C, Hartmann-Boyce J. Different doses, durations and modes of delivery of nicotine replacement therapy for smoking cessation. Cochrane Database Syst Rev. 2019;4:CD013308. doi:10.1002/14651858.cd013308

11. Stead LF, Perera R, Bullen C, et al. Nicotine replacement therapy for smoking cessation. Cochrane Database Syst Rev. 2012;11:CD000146. doi:10.1002/14651858.cd000146.pub3

12. Adams AJ, Hudmon KS. Pharmacist prescriptive authority for smoking cessation medications in the United States. J Am Pharm Assoc. 2018;58(3):253-257. doi:10.1016/j.japh.2017.12.015

13. Bernstein SL, D'Onofrio G, Rosner J, et al. Successful Tobacco Dependence Treatment in Low-Income Emergency Department Patients: A Randomized Trial. Ann Emerg Med. 2015;66(2):140-147. doi:10.1016/j.annemergmed.2015.03.030

14. Lemhoefer C, Rabe GL, Wellmann J, et al. Emergency Department-Initiated Tobacco Control: Update of a Systematic Review and Meta-Analysis of Randomized Controlled Trials. Prev Chronic Dis. 2017;14:E89. doi:10.5888/pcd14.160434

15. Bernstein SL, Bijur P, Cooperman N, et al. A randomized trial of a multicomponent cessation strategy for emergency department smokers. Acad Emerg Med. 2011;18(6):575583. doi:10.1111/j.1553-2712.2011.01097.x

16. Anders ME, Sheffer CE, Barone CP, Holmes TM, Simpson DD, Duncan AM. Emergency department-initiated tobacco dependence treatment. Am J Health Behav. 2011;35(5):546-556. doi:10.5993/ajhb.35.5.4

17. Mahabee-Gittens EM, Ammerman RT, Khoury JC, et al. Healthy families: study protocol for a randomized controlled trial of a screening, brief intervention, and referral to treatment intervention for caregivers to reduce secondhand smoke exposure among pediatric emergency patients. BMC Public Health. 2017;17(1):374. doi:10.1186/s12889-017-4278-8

18. Fiore MC, Jaén CR, Baker TB, et al. Treating tobacco use and dependence: 2008 update. Clinical practice guideline. Rockville, MD: US Department of Health and Human Services. doi:10.1037/e481882008-001 
19. Rigotti NA. Pharmacotherapy for smoking cessation in adults. https://www.uptodate.com/contents/ pharmacotherapy-for-smoking-cessation-in-adults. Accessed January 15, 2020.

20. Vinokur AD, Price RH, Caplan RD. Hard times and hurtful partners: how financial strain affects depression and relationship satisfaction of unemployed persons and their spouses. J Pers Soc Psychol. 1996;71(1):166-179. doi:10.1037/0022-3514.71.1.166

21. Biener L, Abrams DB. The Contemplation Ladder: validation of a measure of readiness to consider smoking cessation. Health Psychol. 1991;10(5):360-365. doi:10.1037/0278-6133.10.5.360

22. Chabrol H, Niezborala M, Chastan E, de Leon J. Comparison of the Heavy Smoking Index and of the Fagerstrom Test for Nicotine Dependence in a sample of 749 cigarette smokers. Addict Behav. 2005;30(7):14741477. doi:10.1016/j.addbeh.2005.02.001

23. Mahabee-Gittens EM, Khoury JC, Ho M, Stone L, Gordon JS. A smoking cessation intervention for low-income smokers in the ED. Am J Emerg Med. 2015;33(8):10561061. doi:10.1016/j.ajem.2015.04.058

24. Enyioha C, Meernik C, Ranney L, Goldstein AO, Sellman $\mathrm{K}$, Kistler CE. Willingness-to-try various tobacco cessation methods among US adult cigarette smokers. Tob Prev Cessat. 2019;5(May). doi:10.18332/tpc/108555

25. Choo EK, Sullivan AF, LoVecchio F, Perret JN, Camargo CA, Boudreaux ED. Patient preferences for emergency department-initiated tobacco interventions: a multicenter cross-sectional study of current smokers. Addict Sci Clin Pract. 2012;7:4. doi:10.1186/1940-0640-7-4

26. Liebmann EP, Scheuermann TS, Faseru B, Richter KP. Critical steps in the path to using cessation pharmacotherapy following hospital-initiated tobacco treatment. BMC Health Serv Res. 2019;19(1):246. doi:10.1186/s12913-019-4059-4

27. Chui CY, Thomas D, Taylor S, et al. Factors associated with nicotine replacement therapy use among hospitalised smokers. Drug Alcohol Rev. 2018;37(4):514-519. doi:10.1111/dar.12661

28. Boudreaux ED, Baumann BM, Friedman K, Ziedonis DM. Smoking stage of change and interest in an emergency department-based intervention. Acad Emerg Med. 2005;12(3):211-218. doi:10.1111/j.1553-2712.2005.tb00871.x

29. McCarthy M, Siahpush M, Shaikh RA, Sikora Kessler A, Tibbits M. Social Disparities in Unaided Quit Attempts Among Daily Current and Former Smokers: Results From the 2010-2011 Tobacco Use Supplement to the Current Population Survey. Nicotine Tob Res. 2016;18(8):17051710. doi:10.1093/ntr/ntw007

30. Sheffer C, Brackman S, Lercara C, et al. When Free Is Not for Me: Confronting the Barriers to Use of Free Quitline Telephone Counseling for Tobacco Dependence. Int J Environ Res Public Health. 2015;13(1):15. doi:10.3390/ijerph13010015
31. Sheffer CE, Brackman SL, Cottoms N, Olsen M. Understanding the barriers to use of free, proactive telephone counseling for tobacco dependence. Qual Health Res. 2011;21(8):1075-1085. doi:10.1177/1049732311404248

32. Reitzel LR, Langdon KJ, Nguyen NT, Zvolensky MJ. Financial strain and smoking cessation among men and women within a self-guided quit attempt. Addict Behav. 2015;47:66-69. doi:10.1016/j.addbeh.2015.03.026

33. Siahpush M, McNeill A, Borland R, Fong GT. Socioeconomic variations in nicotine dependence, selfefficacy, and intention to quit across four countries : findings from the International Tobacco Control (ITC) Four Country Survey. Tob Control. 2006;15(suppl_3):iii71-iii75. doi:10.1136/tc.2004.008763

34. Siu AL, Force USPST. Behavioral and Pharmacotherapy Interventions for Tobacco Smoking Cessation in Adults, Including Pregnant Women: U.S. Preventive Services Task Force Recommendation Statement. Ann Intern Med. 2015;163(8):622-634. doi:10.7326/m15-2023

35. Stefan MS, Pack Q, Shieh MS, et al. The Association of Nicotine Replacement Therapy With Outcomes Among Smokers Hospitalized for a Major Surgical Procedure. Chest. 2019:S0012-3692(0019)34368-34365. doi:10.1016/j.chest.2019.10.054
CONFLICTS OF INTEREST

The authors have completed and submitted the ICMJE Form for Disclosure of Potential Conflicts of Interest and none was reported.

\section{FUNDING}

This research was funded by the National Institutes of Health R01HD083354 (EMMG) and K01DA044313 (ALM).

\section{AUTHORS' CONTRIBUTIONS}

EMMG conceived the study, overall study aims, study design, and wrote the first draft of the paper. MET and JCK conducted the statistical analyses and interpreted the data. LS supervised participant recruitment and data collection and created databases for analyses. ALM and JSG provided input on the overall study aims, analyses and interpretation of data. All authors made critical comments and revised drafts of the paper. All authors read and approved the final manuscript.

\section{PROVENANCE AND PEER REVIEW}

Not commissioned; externally peer reviewed. 